\section{CLINICAL REPORT}

\author{
X.-F. Lv \\ Y.-W. Qiu \\ X.-L. Zhang \\ L.-J. Han \\ S.-J. Qiu \\ W. Xiong \\ G. Wen \\ Y.-Z. Zhang \\ J. Zhang
}

\title{
Primary Intracranial Choriocarcinoma: MR Imaging Findings
}

SUMMARY: PICCC is the rarest, most malignant primary intracranial GCT. The purpose of this study was to describe and characterize the MR imaging findings in a series of 7 patients $(6$ males and 1 female; mean age, 11.9 years) with pathologically proved PICCC in our institution from 2004 to 2009. All tumors were located within the pineal $(n=6)$ or suprasellar $(n=1)$ regions. On T2-weighted MR imaging, the lesions appeared markedly heterogeneous with areas of both hypointensity and hyperintensity reflecting the histologic heterogeneity, including hemorrhage, fibrosis, cysts, or necrosis. Heterogeneous $(n=7)$, ringlike $(n=4)$, and/or intratumoral nodular $(n=3)$ enhancement was noted on T1-weighted images with gadolinium. These MR imaging findings, combined with patient age and serum $\beta$-HCG levels, may prove helpful in distinguishing PICCC from the more common primary brain tumors, thereby avoiding biopsy of this highly vascular tumor.

ABBREVIATIONS: GCT= germ cell tumor; $\mathrm{HCG} / \beta$-HCG $=$ human chorionic gonadotropin/ $\beta$-human chorionic gonadotropin; PICCC = primary intracranial choriocarcinoma

G CTs are uncommon primary tumors of the central nervous system, comprising 3\%-11\% of all intracranial neoplasms in children and $1 \%$ of all primary intracranial neoplasms in adults. ${ }^{1}$ On the basis of histology, they are classified into germinomas, nongerminomatous GCT (teratoma, embryonal carcinoma, endodermal sinus tumor, and choriocarcinoma), and mixed tumors. PICCC, which is characterized by extraembryonic differentiation along trophoblastic lines and high serum and CSF levels of HCG/ $\beta$-HCG, is the rarest and most malignant primary intracranial GCT. ${ }^{2-4}$ The most common sites of occurrence of PICCC are the pineal and suprasellar regions. ${ }^{5,6}$ Intratumoral hemorrhage and extraneural/ CSF metastasis are common manifestations of PICCC, which are responsible for its poor prognosis. ${ }^{2,3}$ A combination of surgery, chemotherapy, and radiation therapy has improved survival. ${ }^{2-4,7,8}$ When the tumor is small enough to allow complete resection, surgery should be considered as the first line of therapy. $4,8,9$

Correct pretreatment diagnosis is important for determining a proper therapeutic plan because PICCCs appear to have a mixed response to treatment. Because patients with PICCC are at high risk for intratumoral hemorrhage and extraneural/ CSF metastasis, biopsy for histologic diagnosis is not recommended. Markedly elevated HCG/ $\beta$-HCG levels are strongly suggestive of choriocarcinomas, though HCG/ $\beta$-HCG elevations can occur with other GCTs containing syncytiotrophoblastic giant cells. ${ }^{3,10}$

Since Askanazy's first report in 1906, ${ }^{11}$ fewer than 110 cases of PICCC have been reported in the literature, ${ }^{3,6}$ and most were case reports. Only a few studies have included a description of the radiologic findings. ${ }^{2,3,6}$ The purpose of this report was to present the MR imaging features in 7 cases of PICCC. To our knowledge, our series is the largest collection of PICCC cases with MR imaging to date.

Received March 21, 2010; accepted after revision May 2.

From the Department of Medical Imaging Center, Nan Fang Hospital, Southern Medical University, Guangzhou, China.

Please address correspondence to Xuelin Zhang, Prof., Department of Medical Imaging Center, Nan Fang Hospital, Southern Medical University, Guangzhou, China; e-mail: zhangxuelin_2010@163.com

DOl 10.3174/ajnr.A2194

\section{Materials and Methods}

From 2004 to 2009, 7 consecutive patient records with histologically proved PICCC were reviewed after receiving approval from the institutional review board at our hospital. The 7 patients included 1 female and 6 males, ranging in age from 7 to 19 years, with a mean age of 11.9 years. The diagnosis of PICCC relied on the presence of syncytiotrophoblasts without other elements of GCTs either by microscopic findings or immunochemistry. Serum $\beta$-HCG levels were measured in 6 of the 7 patients. In addition, sonographic examination or pelvic CT was performed to exclude the ovaries, uterus, or testicles as the primary site. A retrospective analysis by using medical records and MR imaging findings was performed to evaluate the clinical and MR imaging features of PICCC.

MR imaging was performed in all 7 patients, and CT was performed in 3 patients. MR imaging was performed by using a 1.5T ( $n=$ 5, Magnetom VP; Siemens, Erlangen, Germany) or 3T ( $n=2$, Signa Excite; GE Healthcare, Milwaukee, Wisconsin) scanner. Pregadolinium T1-weighted (TR, 450-600 ms; TE, 12-16 ms) images, T2weighted (TR, 4500-5500 ms; TE, 100-120 ms) images, and gadolinium-enhanced $(0.1-0.2 \mathrm{mmol} / \mathrm{kg}) \mathrm{T} 1$-weighted images were acquired. CT scans (3 patients) were obtained with a Somatom Plus 4 (Siemens). Noncontrast axial CT images were obtained from the vertex to the skull base by using a 3-mm section thickness, $120 \mathrm{kV}$, and 150-200 mAs.

Two neuroradiologists retrospectively reviewed all the MR images and CT scans for consensus. The MR imaging characteristics were analyzed, with particular attention paid to the location, size, shape, margin, internal architecture, signal intensity, and enhancement pattern of the tumor. The presence of calcification within the lesion was determined on noncontrast CT scans in 3 patients.

\section{Results}

\section{Clinical Features}

Most patients had nonspecific symptoms, including headache $(n=6)$ and vomiting $(n=6)$. Other presenting symptoms were blepharoptosis $(n=2)$, nausea $(n=1)$, polydipsia and hyperdiuresis $(n=1)$, cessation of menstruation $(n=1)$, and decreased vision $(n=1)$. The average symptom duration was 3 months and ranged from 2 days to 1 year. Physical signs of precocious puberty, including abnormal weight gain, 

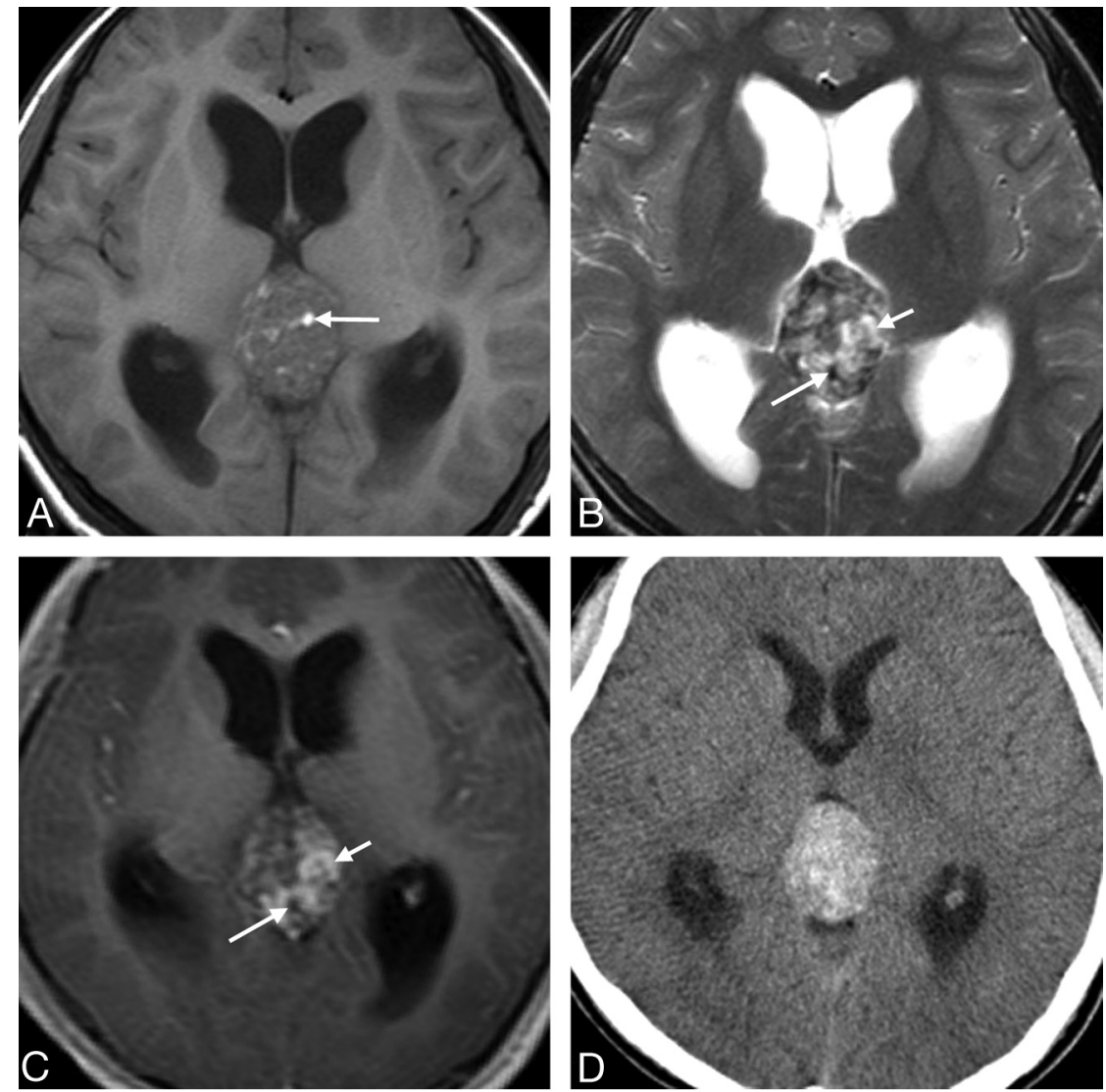

Fig 1. Case 3. Primary intracranial choriocarcinoma of the pineal region in an 11-year-old boy. $A$, Axial T1-weighted image demonstrates an isointense mass with some hyperintense areas (arrow). B, Axial T2-weighted image shows the heterogeneity of the lesion with marked hypointense (longer arrow), isointense, and hyperintense (shorter arrow) areas. C, Postcontrast axial T1-weighted image shows marked heterogeneous enhancement of the isointense and hyperintense areas seen on the T2-weighted image (shorter arrow) and no enhancement of the hypointense areas (longer arrow). D, CT image shows a heterogeneous tumor with hyperattenuation.

growth in the stature, or development of pubic hair, were seen in 2 patients. Preoperative serum $\beta$-HCG levels were measured in 6 patients, and all were markedly elevated (range, 5498-85,303 IU/L; mean, 24,623.3 IU/L; normal range of serum, $<5.0 \mathrm{IU} / \mathrm{L}$ ). Surgical removal ( 5 total resections and 2 subtotal resections) was performed in all patients, and additional chemotherapy $(n=5)$ or radiation therapy $(n=1)$ or both were provided, except for 2 patients who were in very poor general condition. After the initial resection, the postoperative follow-up period for the 7 patients ranged from 1 to 41 months (median, 3 months; mean, 15.6 months). Three patients died after a follow-up of only 1-2 months. One of these 3 patients showed multiple small foci of metastatic choriocarcinoma in the lung as determined by CT accompanied by markedly elevated serum and CSF $\beta$-HCG levels (serum $\beta$-HCG, 8563 IU/L; CSF $\beta$-HCG, 4568 IU/L). Four patients are still alive and have been followed from 3 to 41 months (mean, 26 months) after surgery. Three of the 4 surviving patients had serum HCG levels lower than 10.0 IU/L and MR imaging that showed no sign of recurrence or metastasis. The remaining survivor had 2 small metastatic nodules in the left lateral ventricle on $\mathrm{CT}$.

\section{MR Imaging Findings}

Six of 7 lesions were located in the pineal region (Figs 1 and 2), and 1 was in the suprasellar region (Fig 3). The tumors compressed the tectal plate anteroinferiorly $(n=6)$ and/or pro- truded into the third ventricle $(n=3)$, leading to mild-tomoderate associated hydrocephalus. The tumors ranged in size from 2.0 to $4.5 \mathrm{~cm}$ in greatest diameter, with a mean diameter of $3.1 \mathrm{~cm}$. Six lesions were ovoid $(n=5)$ or round $(n=$ 1 ) with relatively well-defined margins, and the remaining lesion presented as an irregular mass with an indistinct margin and involvement of the adjacent dura.

On T1-weighted images, 6 of 7 lesions were isointense with additional small foci of hyperintensity, and the remaining lesion showed mixed hypointense and isointense signal intensity. In contrast, all 7 lesions were markedly heterogeneous (with areas of both hypointense and hyperintense signal intensity) on T2-weighted images, with additional foci of isointensity in 2 patients (Fig $1 B$ ). Contrast-enhanced MR images demonstrated areas of mild $(n=1)$ or marked $(n=6)$ heterogeneous enhancement (Figs 1-3), ringlike enhancement ( $n=4$, Figs 2 and 3), and/or intratumoral nodular enhancement $(n=3$, Fig 3). On T2-weighted images, enhancement occurred approximately at the isointense or slightly hyperintense areas $(n=5$, Figs $1 B$ and $2 B)$ and hypointense areas $(n=$ 2 , Fig $3 B$ ). Some striplike or patchy areas of hypointensity on T2-weighted images demonstrated no marked contrast enhancement (Figs 1 and 2). Cystic or necrotic areas were seen in all tumors, ranging from several millimeters to several centimeters. Most $(n=6)$ of the tumors had mild-to-moderate peritumoral edema.

On unenhanced CT scans ( $n=3$; patients 1,3 , and 6$)$, all 3 

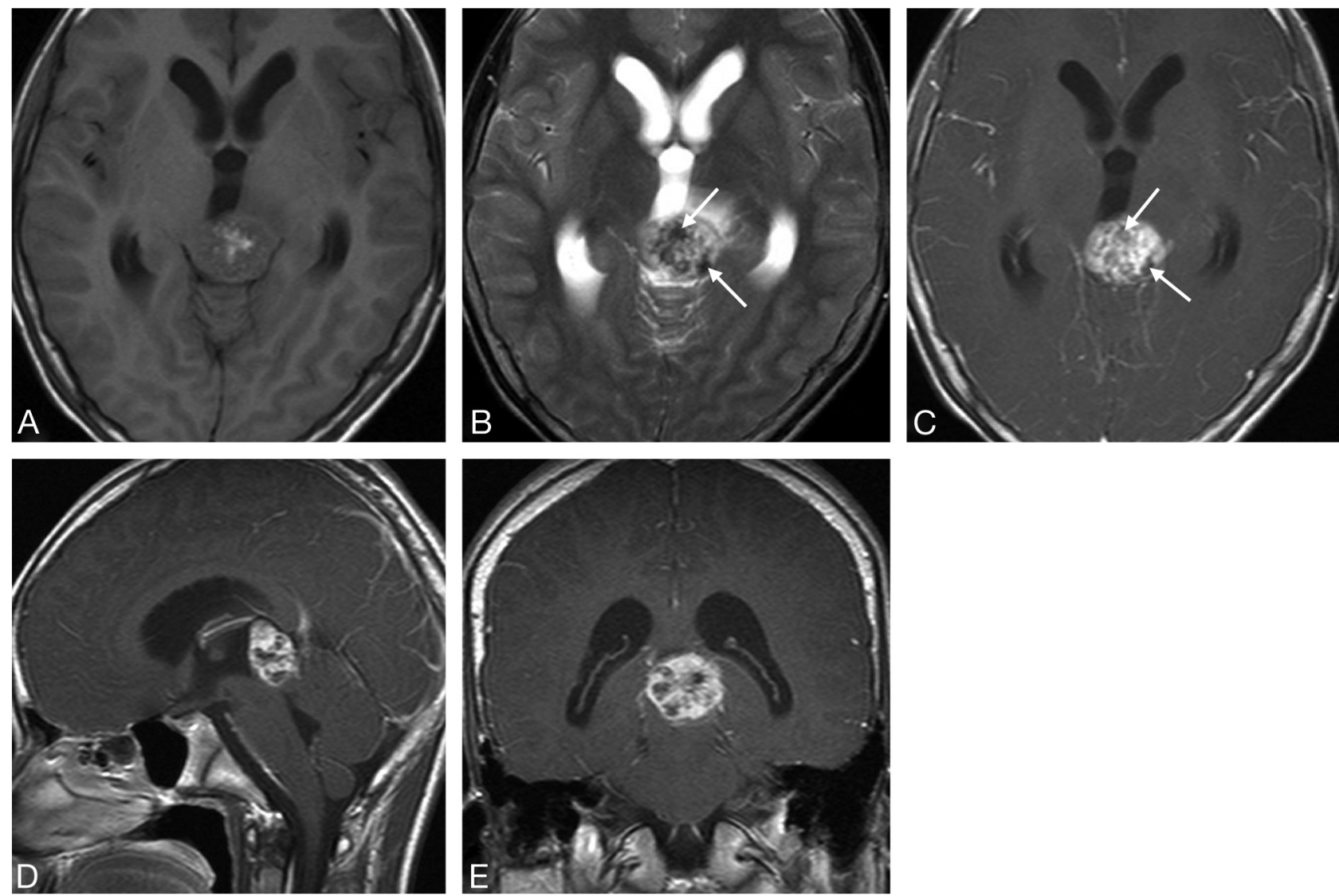

Fig 2. Case 5. Primary intracranial choriocarcinoma of the pineal region in a 14-year-old boy. A, Axial T1-weighted image shows an isointense mass, with hyperintense foci in the center. $B$, Axial T2-weighted image shows a mass with mixed hyperintense and marked hypointense areas (arrows). $C-E$, Postcontrast T1-weighted images show the mass with heterogeneous $(C-E)$ and ringlike enhancement $(D$ and $E)$ and marked hypointense areas on the T2-weighted image without enhancement $(C$, arrows).

lesions showed heterogeneous hyperattenuation (Fig 1D). Only 1 lesion contained punctate calcifications.

\section{Pathology Findings}

Microscopic examination revealed that the resected tumor cells in all patients were dimorphic and were composed of mononucleated trophoblastic cells with uniform round nuclei and clear cytoplasm admixed with large multinucleated syncytiotrophoblastic cells with abundant amphophilic-toeosinophilic cytoplasm. These 2 cell components were intimately admixed and were often associated with areas of hemorrhage, necrosis, fibrosis, and neovascularization. No other germ cell components were present.

\section{Discussion}

Choriocarcinoma is a highly malignant neoplasm composed of biphasic cellular components of mononuclear cytotrophoblasts and multinucleated syncytiotrophoblasts. It usually develops within or outside the uterus following a gestational event, referred to as gestational choriocarcinoma. Nongestational choriocarcinoma can arise from germ cells in gonadal or extragonadal midline locations (mediastinum, retroperitoneum, or pineal gland) and is always mixed with other elements of GCTs. ${ }^{12}$ PICCC belongs to the category of primary intracranial GCTs and is the most malignant of all primary intracranial GCTs. The incidence of PICCC is quite rare, with an estimated ratio of $3 \%-5 \%{ }^{9,13,14}$ among all intracranial GCTs, similar to that in our series of $2.8 \%$. A relatively high incidence of PICCC has been reported in Asia, especially in the Japanese population. A statistical analysis published by the Committee of Brain Tumor Registry of Japan demonstrated that the incidence of PICCC was 3.2\% of all 1127 primary intracranial GCTs. ${ }^{13}$

Clinically, PICCC presents between 3 and 22 years of age (mean age, 11.8 years) and has a male predominance (maleto-female ratio, 74:19). ${ }^{6}$ In the present study, our patients' ages ranged from 7 to 19 years (mean, 11.9 years), and there was also a predilection for males, with the male-to-female ratio of 6:1. The patients' clinical presentations were nonspecific, depending on the location of the tumors. Precocious puberty, which results from the production of HCG, is the most common specific symptom of PICCC occurring in males, especially in patients younger than 12 years of age, and our findings were in accordance with this observation. ${ }^{3,15,16}$

Various sites of PICCC have been reported, including the pineal region ${ }^{3,5,17-21}$ and the suprasellar or parasellar region. ${ }^{5,15,22,23}$ Less common sites include the lateral ventricle, ${ }^{2}$ pituitary fossa, ${ }^{24}$ basal ganglia, ${ }^{2,25}$ and septum pellucidum. ${ }^{6}$ In our study, all tumors were located in the pineal (6/7) and suprasellar (1/7) regions. Although several studies have reported CSF dissemination of PICCC at the time of diagnosis, ${ }^{2}$ most of the PICCCs in our series were single lesions and only 1 case was multifocal. This discrepancy is likely due to the location of the lesions in our series, because most (6/7) of our patients' lesions were located in the pineal region, leading to hydro- 

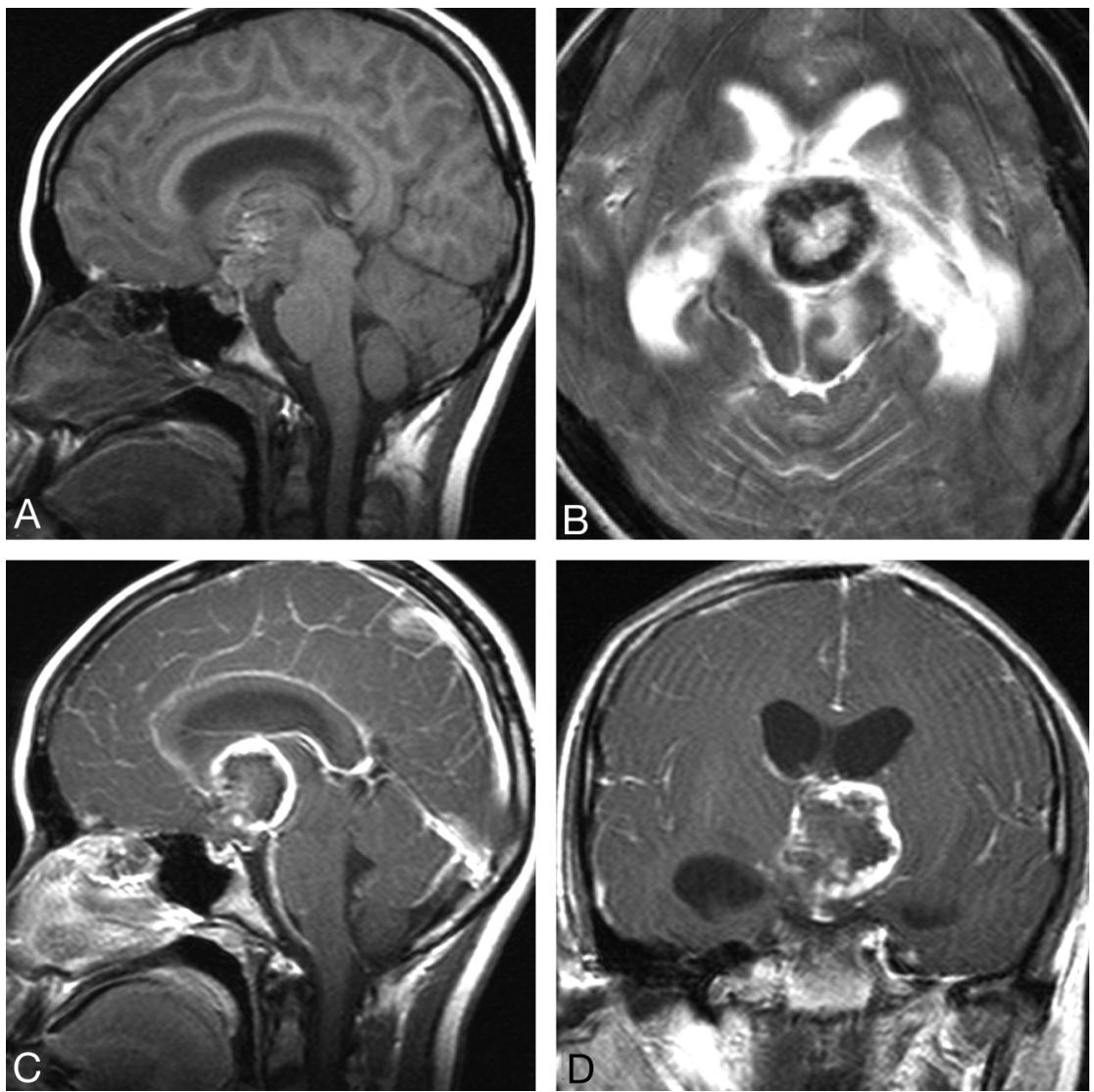

Fig 3. Case 4. Primary intracranial choriocarcinoma of the suprasellar region in a 14-year-old girl. $A$, Sagittal T1-weighted image shows an isointense mass, interspersed with foci of hyperintensity. $B$, T2-weighted image shows a ringlike mass with a solid hypointense rim wall. Sagittal $(C)$ and coronal $(D)$ postcontrast T1-weighted images show ringlike asymmetric thick-rimmed enhancement with mural nodular enhancement.

cephalus, despite the small size of the mass; therefore, they presented at an earlier stage.

Correct pretreatment diagnosis is important for determining a therapeutic plan. HCG/ $\beta$-HCG is a useful biologic tumor marker characteristic of choriocarcinoma. Markedly elevated serum HCG/ $\beta$-HCG levels are strongly suggestive of choriocarcinoma or mixed GCTs with choriocarcinoma elements. In the series described by Matsutani et al, ${ }^{9}$ all patients with choriocarcinoma or mixed GCTs with choriocarcinoma elements had highly elevated levels of serum HCG of $>2000$ IU/L, whereas the serum HCG levels in patients with GCT without choriocarcinoma elements were $<770$ IU/L. In this study, serum $\beta$-HCG levels were assayed in 6 cases, and all demonstrated serum $\beta$-HCG levels $>5000$ IU/L (range, 5498-85,303 IU/L; mean, 24,623.3 IU/L). It appears, therefore, that a patient with a markedly elevated serum $\beta$-HCG level of $>5000$ very likely has choriocarcinoma.

Imaging features aid the pretreatment diagnosis when PICCC is suspected. However, most cases of PICCC reported in the literature are case reports with rather nonspecific radiologic findings. According to previous reports, PICCC is frequently seen as a solitary mass with heterogeneously increased attenuation on noncontrast CT scans. ${ }^{5,18}$ Our CT findings in 3 patients with PICCC were similar to these previous case report findings. Although the presence of calcification has been described in several cases, ${ }^{18}$ it is usually not a dominant feature of PICCC. In our study, only 1 lesion contained punctate calcification in the 3 patients who underwent CT evaluation.
MR imaging has provided improved localization, preoperative evaluation, and characterization of the tumor. In the few published reports, ${ }^{2,3,6,20,23,26}$ the tumor has been described as an ovoid or irregular mass with a large hemorrhagic component on MR imaging. The presence of hemorrhage is a characteristic feature of PICCC. After the administration of contrast, the tumors usually show markedly heterogeneous enhancement. Our results confirm these findings.

In our series, the internal architecture was best displayed on T2-weighted images, in which all lesions displayed heterogeneous hypointensity and hyperintensity. Correlating the MR imaging appearance and the microscopic findings, the heterogeneous signal intensity seen on MR images reflects the various components contained within the lesion, such as intratumoral hemorrhage, fibrosis, cysts, necrosis, or vascular proliferation. Frequent hemorrhage is seen in PICCC due to the fragility of vessels perfusing these trophoblastic tumors and due to the innate capacity of trophoblastic cells to invade and erode vessel walls. Marked striplike or patchy areas of hypointense signal intensity on T2-weighted images corresponded histologically to intratumoral blood products such as hemosiderin and fibrosis; this correspondence was a feature seen in all our patients. On T1-weighted images, these foci of high signal intensity were assumed to correlate with small foci of hemorrhage.

On gadolinium-enhanced T1-weighted images, the heterogeneous, ringlike, and intratumoral nodular enhancement was an additional feature seen in our study. To our knowledge, 
this varying enhancement pattern has not been described previously. Heterogeneous and intratumoral nodular enhancement is most likely due to the sheet- or cordlike arrangement of the trophoblastic cells, which undergo vascular proliferation. The ringlike enhancement may be attributed to plentiful blood vessels on the peripheral rim of the tumor. Consequently, cystic necrosis is more likely to occur in the central area of the tumor. In most of the tumors (5/7), enhancement on T1-weighted images was seen in the isointense or slightly hyperintense areas on T2-weighted images.

Before one suggests the diagnosis of a PICCC, it is important to exclude a hemorrhagic metastasis from the genital system because extracranial choriocarcinoma, especially arising from the gonad, is known to spread via a vascular route. It also often metastasizes to the central nervous system in 3\% 28\% of patients. ${ }^{27}$ The differential diagnosis of a primary intracranial lesion in the pineal or suprasellar region includes other GCTs (germinoma, teratoma, embryonal carcinoma, and endodermal sinus tumor), meningioma, astrocytoma, aneurysm, or venous malformation. The patients' age and biochemical markers, such as serum HCG/ $\beta$-HCG, carcinoembryonic antigen, and $\alpha$-fetoprotein, can be useful in differentiating the lesions from a PICCC. From the standpoint of the MR imaging findings, intratumoral hemorrhage of other GCTs is rare, except for mixed GCTs with a choriocarcinoma element. Although some germinomas may have cystic components, ${ }^{26}$ they demonstrate relatively homogeneous enhancement. Teratomas often show fatty and calcified components. ${ }^{4,20}$ Embryonal carcinoma and endodermal sinus tumor tend be larger than PICCCs and irregular in shape. ${ }^{4}$ In addition, the signal intensity of PICCCs on T1- and T2-weighted images tends to mimic meningiomas, though most PICCCs show heterogeneous enhancement, whereas meningiomas most often have homogeneous enhancement and thickening of the adjacent dura. Astrocytomas tend to show hyperintensity on T2-weighted images, ${ }^{20}$ while PICCCs demonstrate heterogeneous signal intensity with marked hypointense areas. A partially thrombosed aneurysm or venous malformation with blood products demonstrates intrinsic T2 hypointensity and can be excluded on the basis of MR imaging or CT angiographic findings.

The treatment of PICCC remains controversial because the tumor is highly resistant to standard treatment. Although several studies $^{2-4}$ have reported cases of successfully treated PICCCs by using a combination of surgery, chemotherapy, and radiation therapy, the disease is usually fatal and there is no established treatment. Some investigators ${ }^{4,8,9}$ recommend surgery as the first-line therapy when the tumor is small enough to resect completely.

The clinical outcome in patients with PICCC is very poor, which is probably due to their high risk for fatal hemorrhage and their propensity for extraneural/CSF metastasis. Such extraneural metastases usually occur in the lung in one-third or more of patients. ${ }^{16,28}$ The median survival time is 22 months and the 1 - and 2-year survival rates are $61.2 \%$ and $49.8 \%$, respectively. ${ }^{3}$

\section{Conclusions}

Our series of 7 patients with PICCC of the pineal and suprasellar regions revealed that PICCC has characteristic MR im- aging features, such as heterogeneous signal intensity with marked hypointense areas on T2-weighted images. Contrastenhanced T1-weighted images demonstrated heterogeneous, ringlike, or intratumoral nodular enhancement. If a young patient presents with a pineal or suprasellar tumor and imaging features similar to those described above, serum HCG/ $\beta$ HCG levels should be sought and biopsy of these highly vascular tumors avoided.

\section{References}

1. Hoffman HJ, Otsubo H, Hendrick EB, et al. Intracranial germ-cell tumors in children. J Neurosurg 1991;74:545-51

2. Kageji T, Nagahiro S, Matsuzaki K, et al. Successful neoadjuvant synchronous chemo- and radiotherapy for disseminated primary intracranial choriocarcinoma: case report. J Neurooncol 2007;83:199-204

3. Shinoda J, Sakai N, Yano H, et al. Prognostic factors and therapeutic problems of primary intracranial choriocarcinoma/germ-cell tumors with high levels of HCG. J Neurooncol 2004;66:225-40

4. Kyritsis AP. Management of primary intracranial germ cell tumors. J Neurooncol 2010;96:143-49. Epub 2009 Jul 9

5. Page R, Doshi B, Sharr MM. Primary intracranial choriocarcinoma. J Neurol Neurosurg Psychiatry 1986;49:93-95

6. Koyama S, Tsubokawa T, Katayama Y, et al. Choriocarcinoma of the septum pellucidum: case report. Surg Neurol 1991;35:478-82

7. Ogawa K, Toita T, Nakamura K, et al. Treatment and prognosis of patients with intracranial nongerminomatous malignant germ cell tumors: a multiinstitutional retrospective analysis of 41 patients. Cancer 2003;98:369-76

8. Nishizaki T, Kajiwara K, Adachi N, et al. Detection of craniospinal dissemination of intracranial germ cell tumours based on serum and cerebrospinal fluid levels of tumour markers. J Clin Neurosci 2001;8:27-30

9. Matsutani M, Sano K, Takakura K, et al. Primary intracranial germ cell tumors: a clinical analysis of 153 histologically verified cases. J Neurosurg 1997;86:446-55

10. Wolden SL, Wara WM, Larson DA, et al. Radiation therapy for primary intracranial germ-cell tumors. Int J Radiat Oncol Biol Phys 1995;32:943-49

11. Askanazy M. Die Teratom und Chorioepithelioma der Zwirbel. Verh Dtsch Ges Pathol 1906;10:58-76

12. Cheung AN, Zhang HJ, Xue WC, et al. Pathogenesis of choriocarcinoma: clinical, genetic and stem cell perspectives. Future Oncol 2009;5:217-31

13. Report of the brain tumor registry of Japan (1969-1993). Neurol Med Chir 2000;40(suppl):1-106

14. Jennings MT, Gelman R, Hochberg F. Intracranial germ-cell tumors: natural history and pathogenesis. J Neurosurg 1985;63:155-67

15. Fujita K, Obora Y, Fujiwara K, et al. Suprasellar germinoma and primary suprasellar choriocarcinoma: report of ten cases [in Japanese]. No Shinkei Geka 1980;8:819-26

16. Hongo T, Fujii $\mathrm{Y}$, Fukuoka T, et al. Long-term treatment in infantile choriocarcinoma. Acta Paediatr Jpn 1992;34:52-59

17. Hasegawa $\mathrm{H}$, Ushio $\mathrm{Y}$, Hori $\mathrm{M}$, et al. Primary intracranial choriocarcinoma in the pineal region: a case report. Med J Osaka Univ 1974;25:63-71

18. Kohyama S, Uematsu M, Ishihara S, et al. An experience of stereotactic radiation therapy for primary intracranial choriocarcinoma. Tumori 2001;87: $162-65$

19. Blakeley JO, Grossman SA. Management of pineal region tumors. Curr Treat Options Oncol 2006;7:505-16

20. Tien RD, Barkovich AJ, Edwards MS. MR imaging of pineal tumors. AJR Am J Roentgenol 1990;155:143-51

21. Bjornsson J, Scheithauer BW, Leech RW. Primary intracranial choriocarcinoma: a case report. Clin Neuropathol 1986;5:242-45

22. Lawton CT, Deveikis J, Rumboldt Z, et al. Carotid cavernous fistula in CNS choriocarcinoma. Pediatr Blood Cancer 2008;50:893-95

23. Sievers EL, Berger M, Geyer JR. Long-term survival of a patient with primary sellar choriocarcinoma with pulmonary metastases: a case report. Med Pediatr Oncol 1996;26:293-95

24. Furukawa F, Haebara H, Hamashima Y. Primary intracranial choriocarcinoma arising from the pituitary fossa: report of an autopsy case with literature review. Acta Pathol Jpn 1986;36:773-81

25. Kurisaka M, Moriyasu N, Kitajima K. Immunohistochemical studies of brain tumors associated with precocious puberty: a preliminary report of correlation between tumor secreting hormone and Leydig cells in precocious puberty. Neurol Med Chir (Tokyo) 1979;19:675-82

26. Liang L, Korogi $\mathrm{Y}$, Sugahara T, et al. MRI of intracranial germ-cell tumours. Neuroradiology 2002;44:382-88

27. Suresh TN, Santosh V, Shastry KV, et al. Intracranial haemorrhage resulting from unsuspected choriocarcinoma metastasis. Neurol India 2001;49:231-36

28. Yamagami T, Handa H, Takeuchi J, et al. Choriocarcinoma arising from the pituitary fossa with extracranial metastasis: a review of the literature. Surg Neurol 1983;19:469-80 